\title{
Síndrome antifosfolípidos catastrófico. Reporte de caso y revisión bibliográfica
}

\author{
Luis del Carpio-Orantes ${ }^{1}$, Chantall Citlally Anaya-Martínez y Elías Bonilla-Casas ${ }^{2}$ \\ ${ }^{1}$ Unidad de Cuidados Intensivos; '2Servicio de Cirugía General. Hospital General de Zona No. 71, Delegación Veracruz Norte, Instituto Mexicano \\ del Seguro Social, Veracruz, Ver., México
}

\section{Resumen}

Se reporta un caso de una condición clínica sumamente rara, la cual cursa con falla multiorgánica aguda posterior a una tormenta trombótica relacionada con anticuerpos antifosfolípidos, el denominado síndrome antifosfolípidos (SAF) catastrófico, el cual comenzó como un cuadro recurrente de trombosis mesentérica, con antecedentes de insuficiencia venosa y úlceras distales probablemente asociadas a un SAF no identificado, ameritando manejo en terapia intensiva y la consulta por el experto mundial Dr. Ricard Cervera, quien confirmó el diagnóstico y recomendó tratar como tal. La evolución del paciente fue satisfactoria hasta el momento. Se hacen recomendaciones finales de diagnóstico y se comentan las opciones de tratamiento actuales, como rituximab o eculizumab. El presente caso fue agregado al registro internacional de SAF catastrófico (International CAPS Registry), que actualmente alberga alrededor de 500 casos en todo el mundo.

PALABRAS CLAVE: Síndrome antifosfolípidos catastrófico. Trombosis mesentérica. Falla multiorgánica.

\begin{abstract}
The present document is the report of a case of a very rare clinical entity, which presents with acute multiorganic failure after a thrombotic storm related to antiphospholipid antibodies, the so-called catastrophic antiphospholipid syndrome, which began as a recurrent picture of mesenteric thrombosis, with a previous history of venous insufficiency and distal ulcers probably associated with an unidentified antiphospholipid; deserving management in intensive care and the consultation by the world expert, Dr. Ricard Cervera who confirmed the diagnosis and recommend treating as such entity, the patient's evolution was satisfactory so far. Final recommendations for diagnosis and current treatment options such as rituximab or eculizumab are made. The present case was added to the international registry that currently houses around 500 cases worldwide (International CAPS Registry).
\end{abstract}

KEY WORDS: Catastrophic antiphospholipid syndrome. Mesenteric thrombosis. Multiorgan failure.

\section{Correspondencia:}

Luis del Carpio-Orantes

Prol. Av. Salvador Díaz Mirón, s/n

Floresta

Fecha de recepción: 16-12-2016

Fecha de aceptación: 26-04-2016

C.P. 91940, Veracruz, Ver., México

E-mail: Neurona23@hotmail.com

DOI:10.24875/GMM.17003124
Gac Med Mex. 2017;153:531-536

Contents available at PubMed www.gacetamedicademexico.com 


\section{Presentación del caso}

Se presenta el caso de un varón de 52 años de edad con antecedentes de úlceras vasculares periféricas y síndrome posflebítico crónico (Fig. 1), quien ingresó por cuadro de dolor abdominal agudo presentando además íleo, neumatosis intestinal y líquido libre evidenciados por tomografía abdominal (Figs. 2 a 4). Es sometido a laparotomía exploradora y se encuentra necrosis intestinal, necrosis del omento y hemoperitoneo de $1800 \mathrm{cc}$; se consideró secundario a trombosis mesentérica segmentaria y se realizó resección de intestino delgado $(80 \mathrm{~cm})$, omentectomía e ileostomía. El cuadro recurrió, ameritando una segunda intervención quirúrgica, y desarrollando falla multiorgánica (renal, pulmonar, hepática, hematológica [coagulación intravascular diseminada], cardiovascular y probablemente suprarrenal) en menos de 7 días. En esta ocasión se realizó resección de íleo distal por isquemia $(60 \mathrm{~cm})$ e ileostomía. Ingresó en la unidad de cuidados intensivos, donde se le brinda soporte hemodinámico y de ventilación mecánica. Presentó un tercer evento abdominal con necrosis del estoma, que ameritó remodelación del mismo.

Dado lo anterior, se inició protocolo de trombofilias por el cuadro trombótico intestinal recurrente, del cual destacó la presencia de anticuerpos anticardiolipinas IgG e IgM con positividad débil, aunado a evidencia histopatológica de necrosis coagulativa y hemorragia transmural intestinal, y necrosis y congestión vascular en el epiplón. Ante la duda del cuadro, solicitamos la evaluación del Dr. Ricard Cervera, experto mundial en esta enfermedad, quien amablemente comentó que podría tratarse de un cuadro de síndrome antifosfolípidos (SAF) catastrófico y recomendó tratarlo como tal, empleándose heparina de bajo peso molecular (enoxaparina, $1 \mathrm{mg} / \mathrm{kg}$ al día), metilprednisolona en pulsos ( $3 \mathrm{~g} / \mathrm{dí} a)$ y posteriormente dosis de mantenimiento, e inmunoglobulina intravenosa $(0.4 \mathrm{~g} / \mathrm{kg}$ al día por 5 días).

La evolución fue satisfactoria, con remisión de todas las lesiones orgánicas, y la hepática, con remisión en forma lenta y paulatina. Actualmente el paciente se encuentra estable en la unidad de cuidados intensivos, ya sin apoyo ventilatorio ni aminas, y cursa con infecciones asociadas al cuidado de la salud (neumonía, de herida quirúrgica) en vías de control y con miopatía del enfermo grave. Se le ha realizado entero-entero anastomosis por ileostomía previa, con buena tolerancia (Tabla 1).

El paciente fue incluido en el registro internacional de SAF catastrófico (International CAPS Registry). Consideramos que las úlceras y el síndrome posflebítico crónico pueden ser manifestación de un SAF primario nunca estudiado y que tiene correlación con el cuadro catastrófico que el paciente presentó.

\section{Discusión}

El presente caso cumple todos los criterios para considerarse un SAF catastrófico, presentando como evento agudo un cuadro de isquemia intestinal derivando en necrosis recurrente aunado a falla multiorgánica, seguramente por trombosis intestinal y microtrombosis de órganos diana (riñones, hígado, suprarrenal, pulmones), demostrándose por estudio histopatológico datos de necrosis coagulativa, edema y congestión vascular,

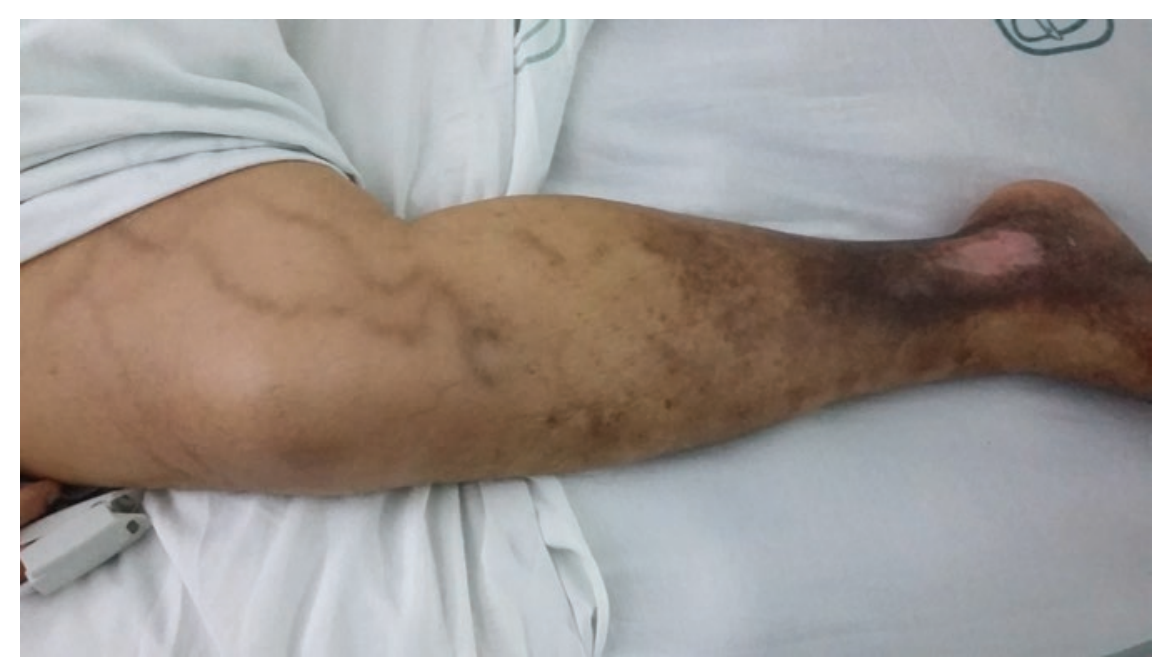

Figura 1. Síndrome posflebítico crónico y úlcera distal cicatricial como manifestaciones de SAF no identificado previamente. 


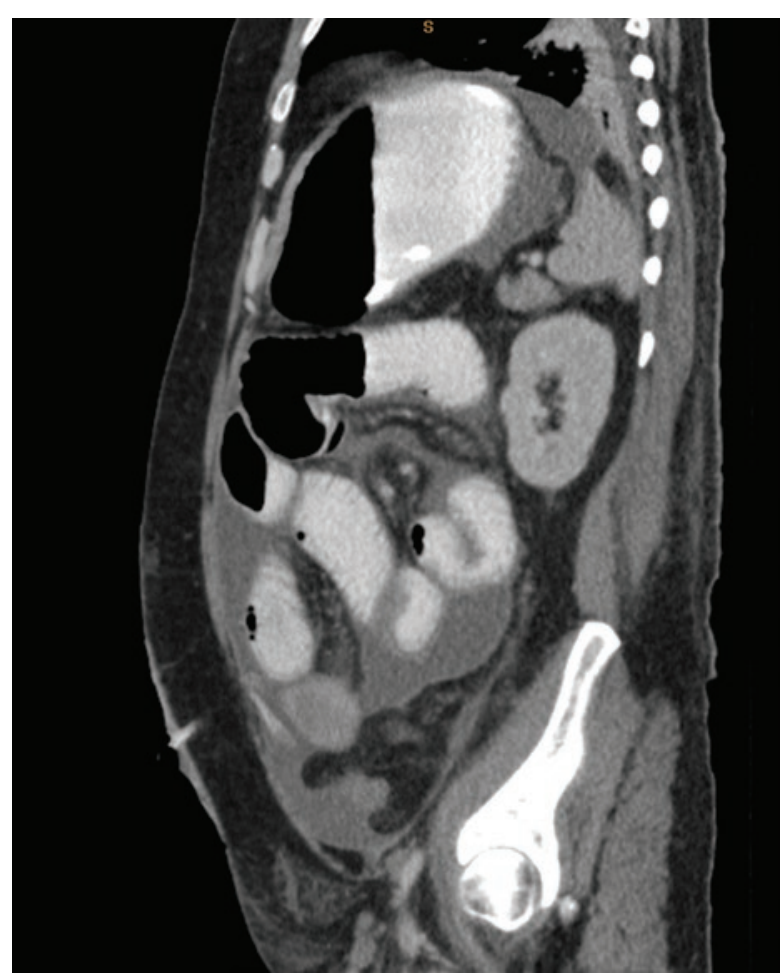

Figura 2. Datos de íleo, líquido libre abdominal y neumatosis intestinal.

así como hemorragia transmural. Durante el estudio de trombosis, por sospecha de trombofilia agregada, se reportaron anticuerpos antifosfolípidos elevados en forma discreta, lo cual se corroboró con una segunda determinación aunado a elevación de otros anticuerpos, relacionados en este caso con anticuerpos extraíbles del núcleo (ENA), reportándose elevación de anti-SSB 0 anti-La, que es una asociación rara en el SAF catastrófico. Consideramos que las lesiones crónicas en las extremidades inferiores del paciente, así como las cicatrices por úlceras y síndrome posflebítico, pueden estar en relación con un SAF primario no estudiado y que fue evidente con esta presentación catastrófica. Ante la duda, por la rareza del diagnóstico, se concertó con el experto mundial, considerando el diagnóstico y recomendando tratar como dicha enfermedad, por lo que optamos por el esquema de heparina no fraccionada, esteroides sistémicos e inmunoglobulina intravenosa, con buena evolución del paciente.

\section{Revisión}

El SAF catastrófico, también denominado síndrome de Asherson, tiene sus primeras descripciones en 1984, pero fue en 1992 cuando el Dr. Ronald A. Asherson (1934-2008) lo describió como una coagulopatía generalizada fuertemente relacionada con los anticuerpos antifosfolípidos, pero totalmente distinta y separada de otras coagulopatías heredadas o adquiridas $^{1,2}$. Dicha definición ha evolucionado y actualmente se le considera una tormenta trombótica con oclusiones microvasculares y macrovasculares en un corto periodo de tiempo, con pobre pronostico pese a recibir tratamiento.

La incidencia se sitúa en un 1\% de todos los casos de SAF, considerándose una variante rara, pero letal, de dicho síndrome. En la última revisión del International CAPS Registry se comentan 500 casos registrados entre 1992 y 2014, agregándose continuamente nuevos casos al registro, como el presente ${ }^{3}$. Respecto del diagnóstico, se han establecido criterios diagnósticos para el SAF catastrófico definido o confirmado, los cuales son:

1) Afectación de tres o más órganos, sistemas 0 tejidos.

2) Presencia de la lesión multiorgánica en menos de 7 días.

3) Confirmación histopatológica de oclusión de vasos pequeños.

4) Presencia de anticuerpos antifosfolípidos.

Se habla de un SAF probable cuando no cumple con todos los criterios anteriores, pero sí puede tener combinaciones que hacen que la probabilidad del síndrome aumente:

- Los cuatro criterios, pero solo afectación de dos órganos, sistemas o tejidos.

- Los cuatro criterios, excepto la presencia de anticuerpos antifosfolípidos.

- Los criterios 1, 2 y 4.

- Los criterios 1, 3 y 4, pero con el desarrollo de un tercer evento entre 8 y 30 días del primer evento, pese a la anticoagulación $n^{4-8}$.

Actualmente, en el último reporte del International CAPS Registry (2016) se aprecia que la mortalidad ha disminuido desde un 50\% (en los primeros reportes) hasta un $33 \%$, probablemente por la mayor identificación del cuadro en sus etapas iniciales y el tratamiento oportuno, aunque se reporta que los casos de SAF catastrófico asociados a lupus eritematoso sistémico tienen la mayor tasa de mortalidad, con un $48 \%$. El sexo más afectado sigue siendo el femenino (69\%), y el grupo de edad más afectado es el de 31-39 años, siendo el promedio de edad a los 38 años. El $60 \%$ de los casos han correspondido a un SAF primario y un $30 \%$ se han asociado a lupus eritematoso sistémico. De los factores predominantes, destacan las infecciones (49\%), la cirugía (17\%) y la malignidad $(16 \%)^{3}$. 
Tabla 1. Características bioquímicas del paciente a su ingreso al hospital, durante la etapa crítica (falla multiorgánica) y en la etapa actual

\begin{tabular}{|c|c|c|c|}
\hline & Ingreso & Etapa crítica (5-7 días) & Etapa actual (50 días) \\
\hline $\mathrm{Hb} /$ leucocitos & $11.3 \mathrm{~g} / 14,900 \mathrm{~mm}^{3}$ & $6.3 \mathrm{~g} / 33,600 \mathrm{~mm}^{3}$ & $8.9 \mathrm{~g} / 9000 \mathrm{~mm}^{3}$ \\
\hline Plaquetas & 206,000 & 10,000 & 117,000 \\
\hline Glucosa & $55 \mathrm{mg}$ & $100 \mathrm{mg}$ & $69 \mathrm{mg}$ \\
\hline Urea/creatinina & $92 / 1.6 \mathrm{mg}$ & $135 / 3.5 \mathrm{mg}$ & $18 / 0.6 \mathrm{mg}$ \\
\hline $\mathrm{Na} / \mathrm{K}^{+}$ & $149 / 3.9 \mathrm{mmol}$ & $128 / 4.5 \mathrm{mmol}$ & $138 / 4.3 \mathrm{mmol}$ \\
\hline Bilirrubina total/BD & $5.2 / 4.5 \mathrm{mg}$ & $14 / 10.2 \mathrm{mg}$ & $9 / 7.6 \mathrm{mg}$ \\
\hline AST/ALT & $75 / 85 U$ & $165 / 148 U$ & $48 / 56 \mathrm{U}$ \\
\hline $\mathrm{DHL}$ & $399 \mathrm{U}$ & $679 \mathrm{U}$ & $330 \mathrm{U}$ \\
\hline TP/TTP & $23.7 / 24.3 \mathrm{~s}$ & $21.8 / 61 \mathrm{~s}$ & $12.8 / 38 \mathrm{~s}$ \\
\hline Fibrinógeno & --- & $167 \mathrm{mg}$ & $440 \mathrm{mg}$ \\
\hline Dímero D & --- & $4,800 \mu \mathrm{g}$ & --- \\
\hline Coombs & --- & Negativo & --- \\
\hline Esquistocitos & --- & Negativo & -- \\
\hline Anticuerpos antinucleares & --- & 0.56 index (negativo) & 0.41 index (negativo) \\
\hline Anticoagulante lúpico & --- & Negativo & Negativo \\
\hline Anticardiolipinas IgG & --- & 11.46 (positivo débil) & 18.2 U/GPL (positivo) \\
\hline Anticardiolipinas IgM & --- & 10.06 (positivo débil) & $<2 \mathrm{U} / \mathrm{MPL}$ \\
\hline Anti-DNA & --- & --- & 17.39 UI (negativo) \\
\hline Anti-SM & --- & -- & 0.31 ratio (negativo) \\
\hline $\mathrm{C} 3 / \mathrm{C} 4 / \mathrm{CH} 50$ & --- & --- & CH50 95.8 U (bajo) \\
\hline Anti-B2 glucoproteína & --- & --- & $6.0 \mathrm{U} / \mathrm{ml}$ \\
\hline Anti-SSA (Ro) & & & 0.71 ratio (negativo) \\
\hline Anti-SSB (La) & & & 1.57 ratio (positivo) \\
\hline Estado clínico & $\begin{array}{c}\text { Abdomen agudo } \\
\text { Síndrome posflebítico }\end{array}$ & $\begin{array}{c}\text { Falla multiorgánica, choque, } \\
\text { coagulación intravascular diseminada, } \\
\text { hipoxemia, ictericia generalizada, } \\
\text { anasarca, encefalopatía }\end{array}$ & $\begin{array}{l}\text { Ictericia en remisión } \\
\text { Miopatía del enfermo grave, } \\
\text { síndrome posflebítico }\end{array}$ \\
\hline
\end{tabular}

Las manifestaciones de falla orgánica más frecuentes son renales $(73 \%)$, pulmonares $(60 \%)$, neurológicas $(56 \%)$, cardiacas $(50 \%)$ y dérmicas $(47 \%)$. Los hallazgos bioquímicos más sobresalientes son la trombocitopenia $(67 \%)$ y la presencia de anticoagulante lúpico (83\%), anticuerpos anticardiolipinas IgG $(81 \%)$ y anticuerpos anti-B2 glucoproteína IgG (78\%). Los anticuerpos antinucleares solo están presente en el 57\%, Ios anti-DNA en el $32 \%$ y los anti-ENA en el $10 \%{ }^{3}$.

En cuanto al tratamiento, se han revisado tratamientos individuales y combinados. De los tratamientos individuales, los más empleados son anticoagulantes
(82\%), esteroides (76\%), plasmaféresis (35\%) e inmunoglobulina intravenosa (27\%). Entre los tratamientos combinados (mayor efectividad, en teoría) destacan los anticoagulantes con esteroides (19\%) y los anticoagulantes + esteroides + plasmaféresis/inmunoglobulina intravenosa $(18 \%)^{9-11}$.

Recientemente se han empleado anticuerpos monoclonales para el tratamiento del SAF catastrófico, pero la evidencia aún es escasa y solo hay reportes de casos anecdóticos. El rituximab fue empleado en 33 pacientes ( $6 \%$ de la serie) y el eculizumab en dos pacientes $(0.2 \% \text { de la serie })^{12}$. Se ha comunicado dos 


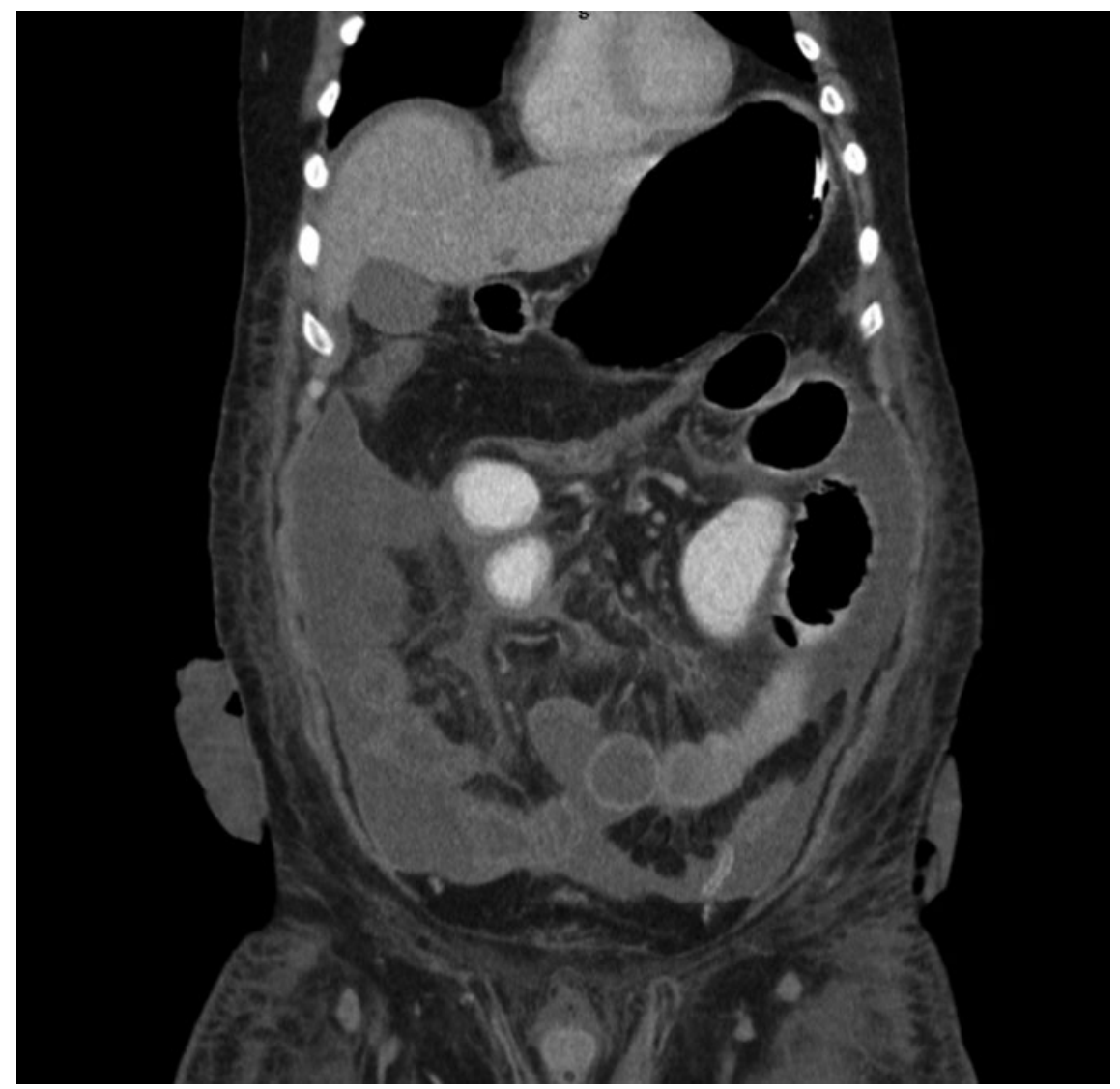

Figura 3. Datos de íleo y neumatosis intestinal, así como distensión de la cámara gástrica.

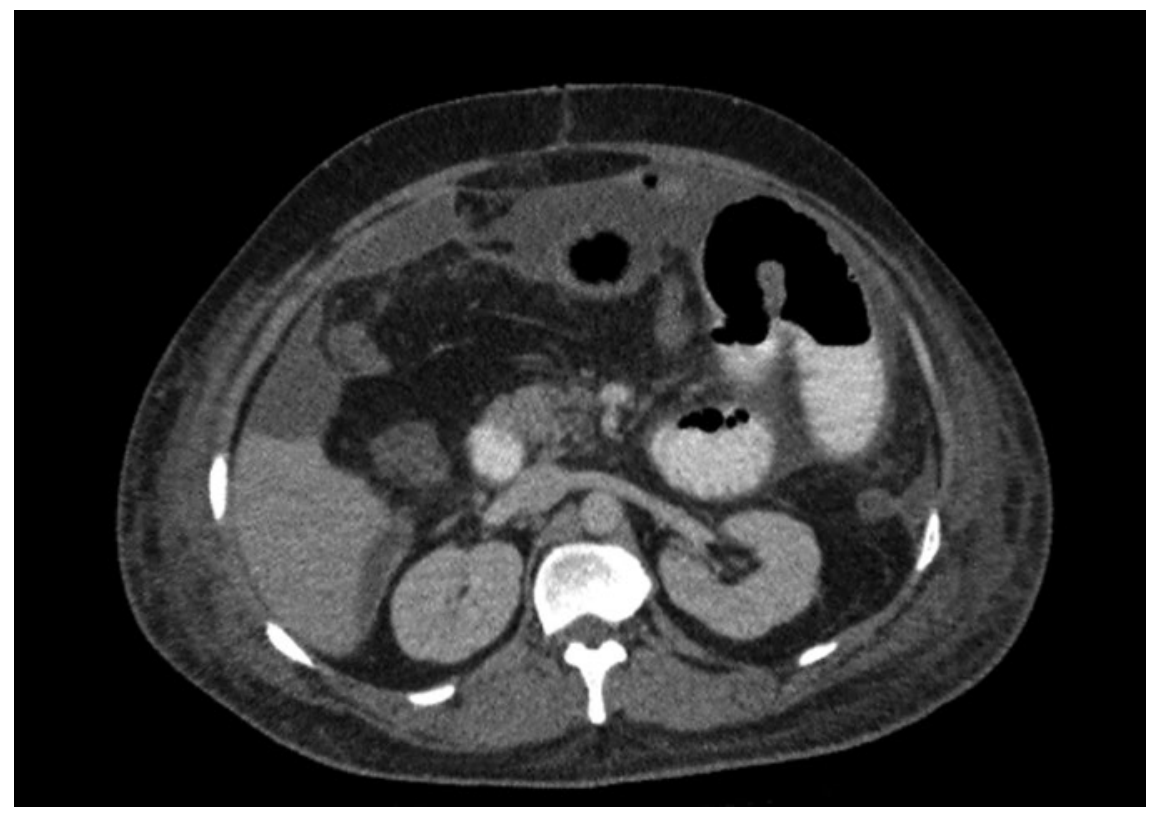

Figura 4. Íleo y neumatosis intestinal como manifestación de trombosis mesentérica.

casos en los cuales se realizó prevención de la trombosis de injertos renales en pacientes con SAF catastrófico, con buen resultado; sin embargo, se recomienda realizar ensayos con mayor cantidad de pacientes. Un reporte de caso empleó eculizumab en una paciente a la que se habían dado esteroides y ciclofosfamida, y persistía el daño renal ameritando hemodiálisis, pero tras el uso del anticuerpo se logró 
prescindir de dicha terapia por mejoría importante de la función renal ${ }^{13}$. De igual forma, otro reporte de caso de un varón demostró mejoría que no se había logrado con anticoagulación, inmunosupresión y recambio plasmático. En conclusión, el eculizumab podría implementarse como preventivo en los pacientes con SAF catastrófico que serán sometidos a trasplante renal y en aquellos en que las terapias convencionales han fallado para controlar la enfermedad, así como en cuadros recurrentes. Acorde a las conclusiones tomadas del International CAPS registry, el rituximab se recomienda para pacientes con SAF catastrófico refractario al tratamiento convencional ${ }^{11-15}$.

Como resumen del tratamiento, todos los pacientes con SAF catastrófico deben ser tratados con anticoagulantes, esteroides y recambio plasmático/inmunoglobulina intravenosa. Los pacientes con SAF catastrófico asociado a lupus eritematoso sistémico deberían ser tratados, además, con ciclofosfamida (Ios pacientes sin lupus pueden empeorar si se les administra ciclofosfamida según la evidencia actual), y para los casos refractarios o recaídas, el emplo de anticuerpos monoclonales como rituximab o eculizumab está plenamente recomendado.

Como factores de mal pronóstico se mencionan la edad joven (principalmente en el grupo de 31-39 años), el sexo femenino y estar asociado a lupus eritematoso sistémico, lo que confiere mayor índice de mortalidad.

\section{Agradecimientos}

Al Dr. Ricard Cervera Segura, Médico Consultor Sénior y Jefe del Servicio de Enfermedades Autoinmunes del Hospital Clínic de Barcelona, Jefe del
Equipo de Enfermedades Autoinmunes Sistémicas del Institut d'Investigacions Biomèdiques August Pi i Sunyer (IDIBAPS) de Barcelona, y Profesor del Departamento de Medicina de la Universidad de Barcelona, donde coordina el Máster Oficial en Enfermedades Autoinmunes, quien siempre estuvo dispuesto a esclarecer nuestras dudas y a orientarnos para la resolución del presente caso.

\section{Bibliografía}

1. Asherson RA. The catastrophic antiphospholipid syndrome. J Rheumatol. 1992;19:508-12.

2. Sciascia S, López-Pedrera C, Roccatello D, et al. Catastrophic antiphospholipid syndrome (CAPS). Best Pract Res Clin Rheumatol. 2012;26:535-41.

3. Rodríguez-Pintó I, Moitinho M, Santacreu I, et al. Catastrophic antiphospholipid syndrome (CAPS): descriptive analysis of 500 patients from the International CAPS Registry. Autoimmun Rev. 2016;15:1120-4.

4. Cervera R, Rodríguez-Pintó I, Colafrancesco S, et al. 14th International Congress on Antiphospholipid Antibodies Task Force Report on Catastrophic Antiphospholipid Syndrome. Autoimmun Rev. 2014;13:699-707.

5. Cervera R. CAPS Registry. Lupus. 2012;21:755-7.

6. Asherson RA, Cervera R, Piette JC, et al. Catastrophic antiphospholipid syndrome. Clinical and laboratory features of 50 patients. Medicine (Balt). 1998;77:195-207.

7. Asherson RA, Cervera R, Piette JC, et al. Catastrophic antiphospholipid syndrome: clues to the pathogenesis from a series of 80 patients. Medicine (Balt). 2001;80:355-77.

8. Aguiar CL, Erkan D. Catastrophic antiphospholipid syndrome: how to diagnose a rare but highly fatal disease. Ther Adv Musculoskelet Dis. 2013;5:305-14.

9. Cervera R, Rodríguez-Pinto I, Espinosa G. Catastrophic antiphospholipid syndrome: task force report summary. Lupus. 2014;23:1283-5.

10. Kazzaz NM, McCune WJ, Knight JS. Treatment of catastrophic antiphospholipid syndrome. Curr Opin Rheumatol. 2016;28:218-27.

11. Tenti S, Cheleschi S, Guidelli GM, et al. Intravenous immunoglobulins and antiphospholipid syndrome: how, when and why? A review of the literature. Autoimmun Rev. 2016;15:226-35.

12. Berman H, Rodríguez-Pintó I, Cervera R, et al. Rituximab use in the catastrophic antiphospholipid syndrome: descriptive analysis of the CAPS registry patients receiving rituximab. Autoimmun Rev. 2013;12:1085-90.

13. Strakhan M, Hurtado-Sbordoni M, Galeas N, et al. 36-year-old female with catastrophic antiphospholipid syndrome treated with eculizumab: a case report and review of literature. Case Rep Hematol. 2014;2014:704371.

14. Shapira I, Andrade D, Allen SL, et al. Induction of sustained remission in recurrent catastrophic antiphospholipid syndrome via inhibition of terminal complement with eculizumab. Arthritis Rheum. 2012;64:2719-23.

15. Lonze BB, Zachary AA, Magro CM, et al. Eculizumab prevents recurrent antiphospholipid antibody syndrome and enables successful renal transplantation. Am J Transplant. 2014;14:459-65. 